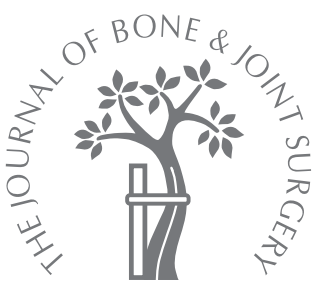

L. B. Solomon, S. A. Callary, A. W. Stevenson, M. A. McGee, M. J. Chehade, D. W. Howie

From Royal Adelaide Hospital and University of Adelaide, Adelaide, Australia

\title{
Weight-bearing-induced displacement and migration over time of fracture fragments following split depression fractures of the lateral tibial plateau
}

A CASE SERIES WITH RADIOSTEREOMETRIC ANALYSIS

We investigated the stability of seven Schatzker type II fractures of the lateral tibial plateau treated by subchondral screws and a buttress plate followed by immediate partial weightbearing. In order to assess the stability of the fracture, weight-bearing inducible displacements of the fracture fragments and their migration over a one-year period were measured by differentially loaded radiostereometric analysis and standard radiostereometric analysis, respectively. The mean inducible craniocaudal fracture fragment displacements measured $-0.30 \mathrm{~mm}(-0.73$ to 0.02$)$ at two weeks and $0.00 \mathrm{~mm}(-0.12$ to 0.15$)$ at 52 weeks. All inducible displacements were elastic in nature under all loads at each examination during follow-up. At one year, the mean craniocaudal migration of the fracture fragments was $-0.34 \mathrm{~mm}(-1.64$ to 1.51$)$.

Using radiostereometric methods, this case series has shown that in the Schatzker type II fractures investigated, internal fixation with subchondral screws and a buttress plate provided adequate stability to allow immediate post-operative partial weight-bearing, without harmful consequences.

Split depression fractures of the lateral tibial plateau (Schatzker type II fractures) ${ }^{1}$ can be treated by subchondral screws passed through a peri-articular plate, or used in conjunction with an antiglide plate, which provides better depression stiffness than the classic L-shaped buttress plate alone. ${ }^{2}$ The former techniques are gaining in popularity. ${ }^{3}$

At present there is no consensus as to the weight-bearing regime that should be followed after fixation of fractures of the tibial plateau. Recommendations vary from non-weight-bearing for 12 to 16 weeks, ${ }^{4}$ nonweight-bearing for six to eight weeks, ${ }^{5}$ nonweight-bearing prescribed on an individual basis $^{6}$ or immediate partial weight-bearing for all patients. ${ }^{7,8}$ Encouraged by the results reported for patients who underwent immediate post-operative partial weight-bearing, ${ }^{7,8}$ our institution has adopted this rehabilitation strategy. However, there are no data on the effects of weight-bearing on the stability of the fracture, or on how early partial weightbearing might affect the stability over time.

For tibial plateau fractures, it has been reported that reduction and the maintenance of reduction of the articular surface to within
$2 \mathrm{~mm}$ in the craniocaudal direction is anatomical. ${ }^{7,9}$ A residual craniocaudal displacement of the articular surface within $3 \mathrm{~mm}$ has also been shown not to influence the outcome. ${ }^{10,11}$ These estimates have been based on manual measurements on plain radiographs, ${ }^{10,11}$ which lack the sensitivity and accuracy to detect fracture migration $<5 \mathrm{~mm},{ }^{12-15}$ and so may be unreliable. In contrast, radiostereometric analysis (RSA) can detect migration of the fragments of a tibial plateau fracture with an accuracy of $\pm 37 \mu \mathrm{m}$ and a precision of $\pm 16 \mu \mathrm{m},{ }^{16}$ and therefore an overall error of within $100 \mu \mathrm{m}$. In addition, RSA can detect rotation of a tibial plateau fracture fragment with an accuracy of $\pm 0.123^{\circ}$ and a precision of $\pm 0.024^{\circ},{ }^{16}$ and hence an overall error of within $0.3^{\circ}$. Another advantage is that by using differentially loaded RSA, ${ }^{17}$ fracture fragment movement under load, or inducible displacement, can also be measured in vivo. In this way, RSA studies on small numbers of patients can accurately monitor displacement of fracture fragments under load and migration during healing. Such investigations have the potential to identify improvements in fracture fixation, and also to define safe rehabilitation protocols. 


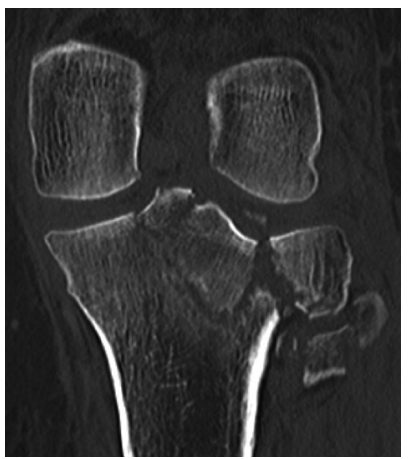

Fig. 1a

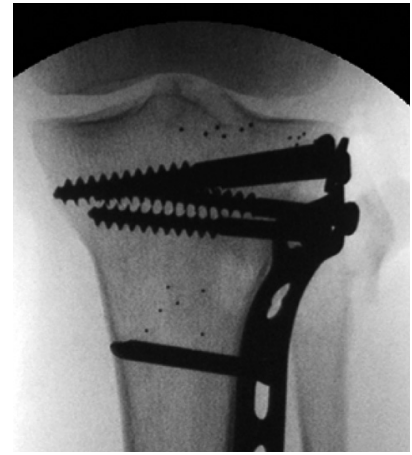

Fig. 1b
A pre-operative coronal CT scan (a) and an intra-operative anteroposterior radiograph (b) showing a split depression fracture of the lateral tibial plateau treated by open reduction and internal fixation using a raft of screws and buttress plate fixation.

To date there are no published studies using RSA to investigate the stability of tibial plateau fractures treated by modern methods of fixation. We hypothesised that in Schatzker type II fractures stabilised with subchondral screws and a buttress plate, early weight-bearing did not compromise the stability of the fracture, and that the reduction achieved at the time of surgery would be maintained. The primary aim was to investigate this using differentially loaded RSA in order to measure inducible displacement of fracture fragments under weight-bearing load, and standard RSA to measure migration of the fracture fragments over time. Our secondary aim was to compare the maintenance of fracture reduction over time, assessed using RSA, with results obtained using manual measurements on standard radiographs, as currently used in clinical practice.

\section{Patients and Methods}

Between September 2007 and July 2008, the stability of depressed fracture fragments was measured during the course of healing in patients treated at our institution for a split depression fracture of the lateral tibial plateau (Schatzer type II). Of the ten patients treated during this period, seven agreed to participate in the study and were compliant with the post-operative weight-bearing protocol. There were two women and five men, with a mean age of 39 years (25 to 58). Institutional Ethics Committee approval was obtained for the investigation.

All the patients underwent open reduction and internal fixation of the fracture, performed through an anterolateral approach, and all of the fractures had a central depression of the lateral tibial plateau of $>1 \mathrm{~cm}$. Based on radiological assessment, macroscopic appearance and physical characteristics during surgery, bone quality of the proximal tibia was considered to be normal in all patients. Depending on the size of the depressed fragments following their reduction, between two and four subchondral screws were used above an L-shaped buttress locking plate (Synthes Inc., West Chester, Pennsylvania) without bone graft, and all the operations were performed by one surgeon (LBS). Between six and nine tantalum beads (RSA Biomedical, Umeå, Sweden) of either $0.8 \mathrm{~mm}$ or $1.0 \mathrm{~mm}$ diameter were inserted into the largest central depressed osteochondral fragment and into the adjacent intact tibial metaphysis ${ }^{16}$ (Fig. 1).

Post-operatively, patients were instructed to partially weight-bear up to $20 \mathrm{~kg}$ using crutches for six weeks, and to perform active range of movement exercises of the knee. They were then instructed to progress to full weightbearing over the next six weeks. Radiographs for RSA were performed for all seven patients within two days of surgery and then at two, six, 12, 18, 26 and 52 weeks, and radiographs for differentially loaded RSA were performed at two, six, 12, 18, 26 and 52 weeks in six patients. One patient was unable to balance safely on her own at two weeks so could not participate in differentially loaded RSA at this stage, but participated at the other timepoints. Standard anteroposterior and lateral radiographs of the knee were also taken post-operatively and at six, 12, 18, 26 and 52 weeks. In addition, long-leg standing radiographs were taken at 52 weeks. ${ }^{18,19}$

For the RSA and differentially loaded RSA examinations, a uniplanar RSA set-up was used with two radiographic tubes. ${ }^{17}$ A room-mounted unit (Philips Bucky Diagnost; Philips Healthcare, Andover, Massachusetts) and a mobile radiographic unit (Philips Practix 8000; Philips Healthcare) were positioned with a $30^{\circ}$ angle between the tubes. The calibration cage (Cage 43; RSA Biomedical) contained two $35 \mathrm{~cm} \times 43 \mathrm{~cm}$ high-resolution digital radiographic cassettes with a focal length to the film of $1.5 \mathrm{~m}$ for each. The radiographic tubes were exposed simultaneously at $60 \mathrm{Kv}$ and $10 \mathrm{mAs}$. The exposures were digitised with an AGFA Centricity CR SP1001 processor (AGFA Healthcare, Mortsel, Belgium). Radiographs were analysed using RSA software (UmRSA version 6.0 and UmRSA DICOM link; RSA Biomedical) by an author (SAC).

Radiographs for differentially loaded RSA were taken with each patient standing on a custom-built platform that allowed the knee to be centred over the calibration cage. During this examination, a digital foot scale was used to measure the load applied during weight-bearing. Differentially loaded RSA radiographs were taken with the foot resting on the scale and applying no load (pre-load), followed by radiographs with the patient applying maximum vertical weight-bearing load on the scale as tolerated without pain (loaded) (Table I). A final pair of radiographs was taken while no load was applied (post-load). ${ }^{17}$ Parallel bars were used by patients for support during the examination to enable 'controlled static' weight-bearing. Inducible displacement of fracture fragments was calculated as the difference in position of the fragments between the loaded and the pre-load radiographs. In order to distinguish whether the displacement of the fracture fragments under load was elastic or plastic, ${ }^{20}$ the position of the fragments in the postload radiograph was compared with that in the pre-load radiograph. 
Table I. The recorded weight in kilograms while weight-bearing as tolerated during differentially loaded radiostereometric analysis examinations for the seven patients

\begin{tabular}{|c|c|c|c|c|c|c|}
\hline & \multicolumn{6}{|c|}{ Weeks } \\
\hline & 2 & 6 & 12 & 18 & 26 & 52 \\
\hline \multicolumn{7}{|c|}{ Patient } \\
\hline 1 & 20 & 30 & 70 & 70 & 70 & 70 \\
\hline $2^{*}$ & - & 30 & 50 & 50 & 50 & 50 \\
\hline 3 & 35 & 60 & 60 & 60 & 70 & 70 \\
\hline 4 & 10 & 30 & 60 & 60 & 60 & 60 \\
\hline 5 & 30 & 75 & 60 & 70 & 85 & 90 \\
\hline 6 & 20 & 40 & 70 & 70 & 80 & 80 \\
\hline 7 & 20 & 50 & 50 & 60 & 60 & 60 \\
\hline
\end{tabular}

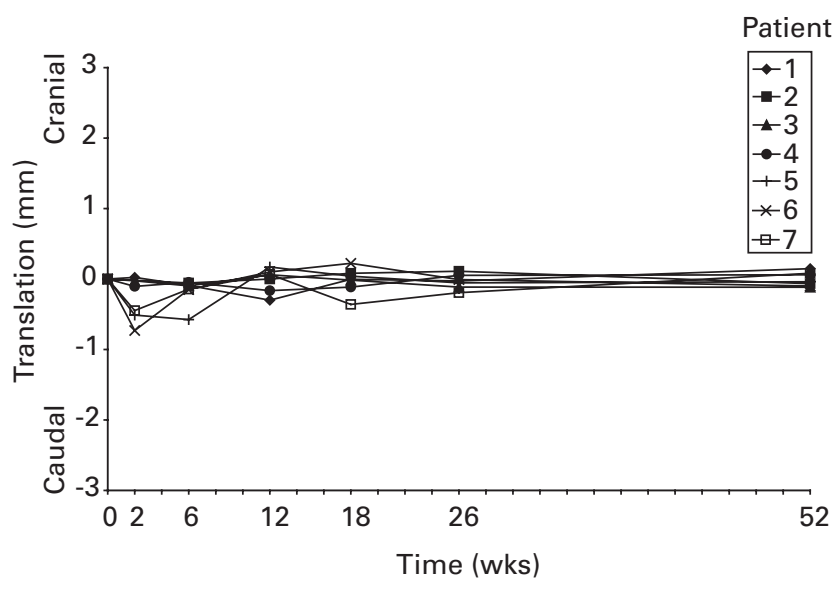

Fig. $2 a$

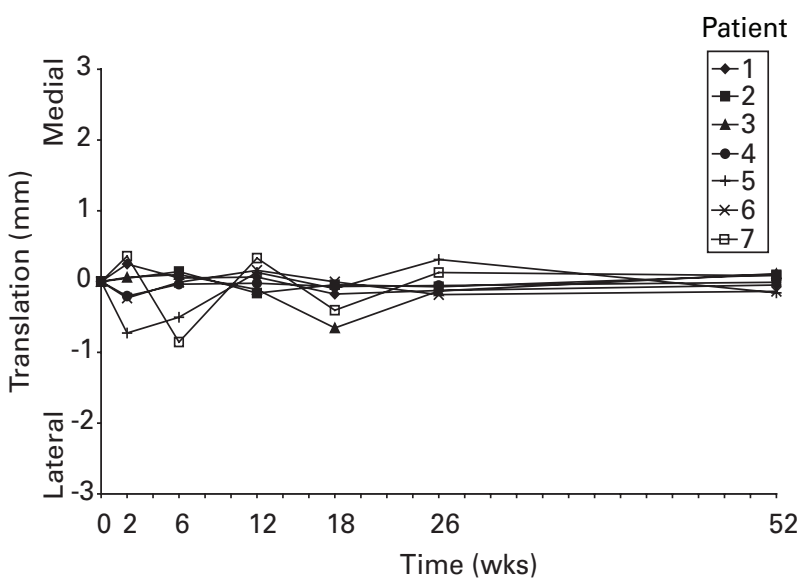

Fig. 2b

Graphs showing the fracture fragment translation under weight-bearing as tolerated at each different time point for each patient in a) the $y$-axis (craniocaudal) and b) the $x$-axis (mediolateral).

In order to measure migration of the central fragment over time, the position of the fracture in the pre-load RSA radiographs taken at each review was compared with that in the initial post-operative RSA radiographs. The direction of induced displacement under load and the migration over time of the fractures were reported as craniocaudal for $y$ axis translations, mediolateral for $x$-axis translations, coronal plane tilt for $z$-axis rotations and sagittal plane tilt for $x$-axis rotations.

Both the differentially loaded RSA and standard RSA examinations identified displacements in each direction of the axes investigated, meaning that some fracture fragments displaced distally whereas others displaced proximally. As both the direction and the magnitude of displacement were thought to be important, the median, range, mean and $95 \%$ confidence interval (CI) were reported for both signed and absolute values.

Maintenance of reduction was also assessed by an author (AWS) using plain radiographs measuring depression of the articular fragments, with $<2 \mathrm{~mm}$ of depression being considered to be anatomical ${ }^{10,11}$ and $<3 \mathrm{~mm}$ to not influence the outcome. ${ }^{10,11}$ Proximal tibial morphology, as a secondary measure of healing, was determined by measurement of change in tibial plateau width ${ }^{10,11}$ and medial and posterior proximal tibial angles. ${ }^{18}$ Clinically acceptable limits for these parameters are an increase in tibial plateau width of $<5 \mathrm{~mm},{ }^{10,11}$ a medial tibial angle between $85.7^{\circ}$ and $88.7^{\circ}$ and a posterior proximal tibial angle between $76.5^{\circ}$ and $83.5^{\circ} .{ }^{18}$

\section{Results}

Radiostereometric measurements of fracture displacement under load. Inducible fracture fragment displacements under controlled static weight-bearing as tolerated revealed that the position of fragments in the post-load examinations was within the error of the method of $100 \mu \mathrm{m}$ from its pre-load position in all cases at all examinations. Therefore, all inducible displacements were elastic.

Inducible translations of the fracture fragments in both craniocaudal and mediolateral directions were $<1 \mathrm{~mm}$ in 


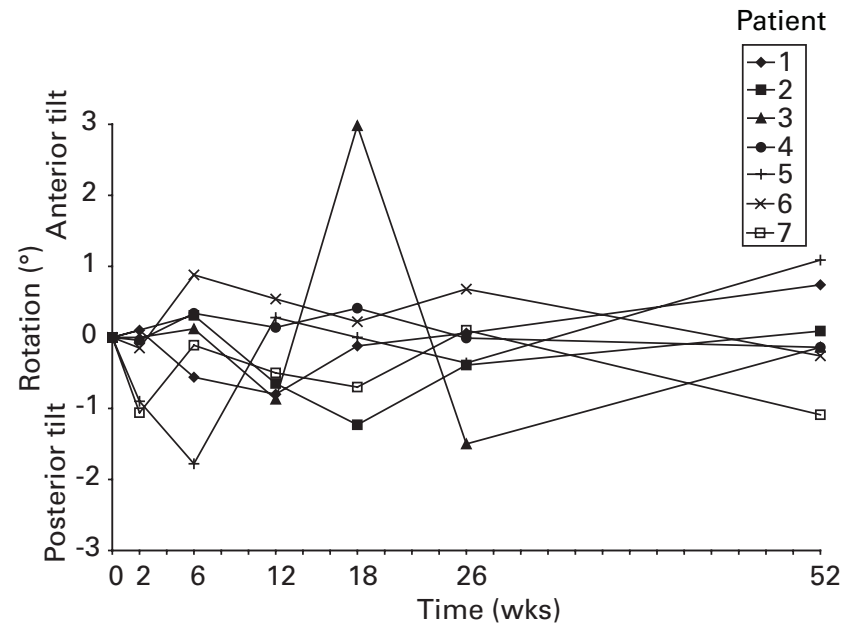

Fig. 3a

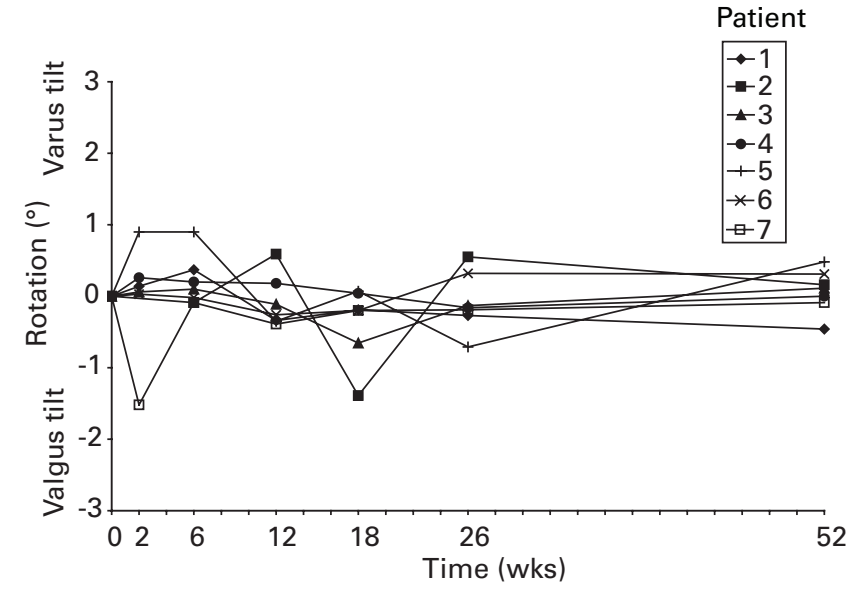

Fig. 3b

Graphs showing the fracture fragment rotation under weight-bearing as tolerated at each different time point for each patient in a) the $x$-axis (anteroposterior tilt), and b) the $z$-axis (varus-valgus tilt).

Table II. Radiostereometric measurements (mean, range) of inducible translation in the craniocaudal and mediolateral directions at two and at 52 weeks

\begin{tabular}{clc}
\hline & \multicolumn{2}{c}{ Mean inducible translation (mm) } \\
\cline { 2 - 3 } & Signed values & Absolute values \\
\hline $\begin{array}{c}\text { Craniocaudal direction } \\
2 \text { weeks }\end{array}$ & $-0.30(-0.73$ to 0.02$)$ & $0.31(0.02$ to 0.73$)$ \\
52 weeks & $0.00(-0.12$ to 0.15$)$ & $0.09(0.04$ to 0.15$)$ \\
Mean difference & & $0.29(0.05$ to 0.63$)$ \\
Mediolateral direction & & \\
2 weeks & $-0.08(-0.73$ to 0.36$)$ & $0.31(0.06$ to 0.73$)$ \\
52 weeks & $-0.01(-0.16$ to 0.11$)$ & $0.09(0.01$ to 0.16$)$ \\
Mean difference & & $0.23(0.05$ to 0.57$)$ \\
\hline
\end{tabular}

all knees at each radiological review (Fig. 2). The mean inducible translations across the knees at two and at 52 weeks using both signed and absolute values are presented in Table II.

Inducible rotations of the fracture fragments under load were $<3^{\circ}$ in the sagittal plane in all knees and $<2^{\circ}$ in the coronal plane (Fig. 3). The mean inducible rotations across the knees at two and 52 weeks using both signed and absolute values are presented in Table III.

Fracture fragment migration over time. At 52 weeks, the central fracture fragments had translated by a mean of $-0.34 \mathrm{~mm}(-1.64$ to 1.51$)$ in the craniocaudal direction and $0.11 \mathrm{~mm}(-2.03$ to 1.35$)$ in the mediolateral direction (Fig. 4, Tables IV and V). The mean coronal plane rotation at 52 weeks was $-2.68^{\circ}\left(-8.58^{\circ}\right.$ to $\left.0.81^{\circ}\right)$ and the sagittal plane rotation was $-0.76^{\circ}\left(-3.48^{\circ}\right.$ to $\left.4.38^{\circ}\right)$ (Fig. 5, Table IV). Using absolute values, at 52 weeks the fracture fragments had migrated by a mean of $0.78 \mathrm{~mm}$ $(0.05$ to 1.64$)$ in the craniocaudal direction and $0.88 \mathrm{~mm}$
Table III. Radiostereometric measurements (mean, range) of inducible rotation in the sagittal and coronal planes at two and at 52 weeks

\begin{tabular}{lll}
\hline & \multicolumn{2}{l}{ Mean inducible rotation $\left(^{\circ}\right)$} \\
\cline { 2 - 3 } & \multicolumn{1}{c}{ Signed values } & Absolute values \\
\hline Sagittal plane & $-0.34(-1.06$ to 0.10$)$ & $0.38(0.00$ to 1.06$)$ \\
2 weeks & $-0.14(-1.09$ to 1.09$)$ & $0.51(0.09$ to 1.09$)$ \\
52 weeks & & $0.20(0.03$ to 1.09$)$ \\
Mean difference & & \\
Coronal plane & & \\
2 weeks & $-0.02(-1.52$ to 0.90$)$ & $0.49(0.03$ to 1.52$)$ \\
52 weeks & $0.07(-0.46$ to 0.48$)$ & $0.23(0.00$ to 0.48$)$ \\
Mean difference & & $0.46(0.05$ to 1.43$)$ \\
\hline
\end{tabular}

(0.06 to 2.03$)$ in the mediolateral direction. The mean absolute rotation at 52 weeks was $2.92^{\circ}\left(0.02^{\circ}\right.$ to $\left.8.58^{\circ}\right)$ in the coronal plane and $2.29^{\circ}\left(0.98^{\circ}\right.$ to $\left.4.38^{\circ}\right)$ in the sagittal plane. Fracture fragment migration had stabilised in five knees by 12 weeks post-operatively. Fracture fragments migrated $>1 \mathrm{~mm}$ between 12 and 26 weeks in one knee, and >1 mm in another between 26 and 52 weeks (Fig. 4).

Manual radiological measurements of fracture migration. All fractures were considered reduced on the first postoperative radiographs and there was $0 \mathrm{~mm}$ of depression of the articular surface at the fracture site at 52 weeks in all cases (Table V). The width of the tibial plateau increased by a mean of $0.86 \mathrm{~mm} \mathrm{(-2} \mathrm{to} 3)$ at 52 weeks. The medial proximal tibial angles at 52 weeks were within normal limits in five of seven tibiae and only just out of normal limits (by $0.3^{\circ}$ ) for two of seven tibiae. The posterior proximal tibial angles were within normal limits in all tibiae. 


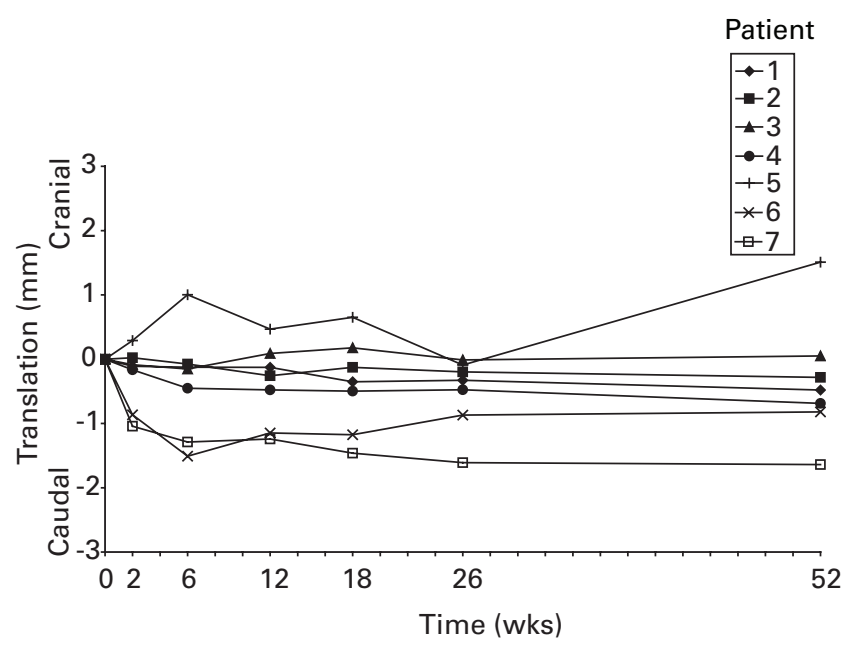

Fig. $4 a$

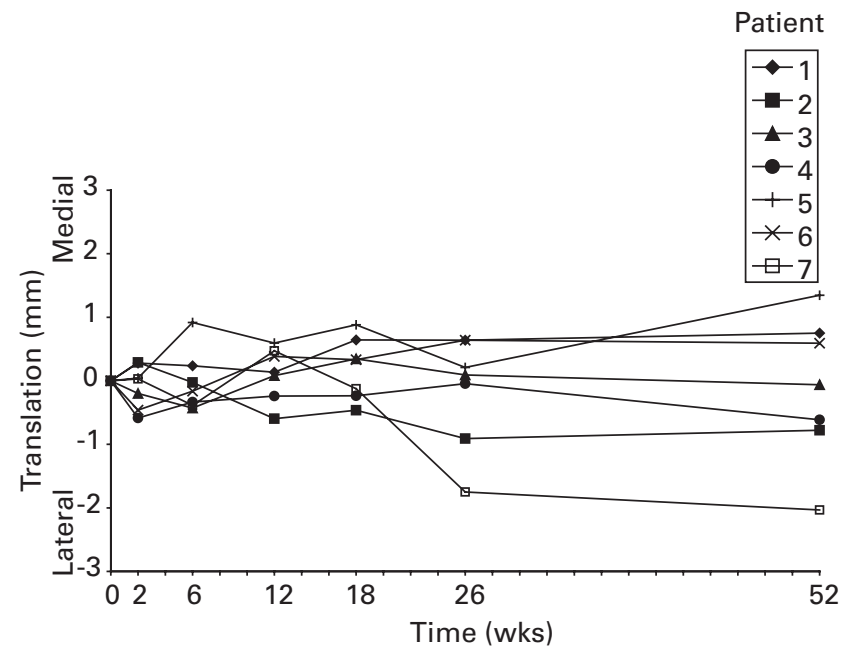

Fig. 4b

Graphs showing the overall fracture fragment translation over time in each patient in a) the $y$-axis (craniocaudal), and b) the $x$-axis (mediolateral).

Table IV. Radiostereometric analysis (RSA) measurements of fracture fragment displacement in the seven patients at 52 weeks

\begin{tabular}{|c|c|c|c|c|}
\hline & \multicolumn{4}{|l|}{ RSA measurements } \\
\hline & Craniocaudal (mm) & Mediolateral (mm) & Varus-valgus tilt $\left(^{\circ}\right)$ & Anteroposterior tilt $\left({ }^{\circ}\right)$ \\
\hline \multicolumn{5}{|l|}{ Patient } \\
\hline 1 & -0.48 & 0.75 & -0.56 & -1.60 \\
\hline 2 & -0.28 & -0.78 & -4.23 & -3.48 \\
\hline 3 & 0.05 & -0.06 & -0.02 & 0.98 \\
\hline 4 & -0.69 & -0.61 & 0.81 & -1.68 \\
\hline 5 & 1.51 & 1.35 & -3.50 & -1.06 \\
\hline 6 & -0.82 & 0.59 & -8.58 & 4.38 \\
\hline 7 & -1.64 & -2.03 & -2.71 & -2.83 \\
\hline Median & -0.48 & -0.06 & -2.71 & -1.60 \\
\hline Mean $\left(95 \% \mathrm{Cl}^{*}\right)$ & $-0.34(-1.23$ to 0.56$)$ & $-0.11(-1.16$ to 0.94$)$ & $-2.68(-5.65$ to 0.28$)$ & $-0.76(-3.23$ to 1.71$)$ \\
\hline Median of absolute values & 0.69 & 0.75 & 2.71 & 1.68 \\
\hline Mean of absolute values $(95 \% \mathrm{Cl})$ & $0.78(0.23$ to 1.33$)$ & $0.88(0.30$ to 1.47$)$ & $2.92(0.18$ to 5.65$)$ & 2.29 (1.08 to 3.49$)$ \\
\hline
\end{tabular}

* $\mathrm{Cl}$, confidence interval

\section{Discussion}

Our investigation using RSA and differentially loaded RSA to measure fracture displacement under load and over time in seven patients with Schatzker type II split depression fractures of the lateral tibial plateau showed that treatment with internal fixation using a raft of subchondral screws and a buttress plate and partial weight-bearing for six weeks successfully maintained the reduction at up to one year. Inducible displacement of fracture fragments cannot be directly measured by any method other than differentially loaded $\mathrm{RSA}^{17}$ or a similar technique using RSA, which has been used to assess movements across the fracture site in the distal radius. ${ }^{21}$ This method of fixation provided sufficient stability to these fractures to allow immediate post-operative partial weight-bearing, thereby offering considerable benefit to the patient in terms of rehabilitation. We anticipate that in the future, single time-point measurements of fracture stability by differentially loaded RSA in the early phases of healing could be used to predict continued stability or impending failure.

We believe that the study provides proof of this principle, but recognise the limitations due to the small number of patients investigated. By showing the potential benefits of RSA and differentially loaded RSA in monitoring tibial plateau fractures in this exploratory case series, we have demonstrated a new area for research in clinical orthopaedics. As yet, we are unable to define the significance of the small migrations of the fracture fragments we recorded. Clinically relevant thresholds for differentially loaded RSA and RSAmeasured displacement over time need to be determined by larger studies, and such findings will need to be examined in relation to the long-term outcomes of these fractures. The other limitation is that the findings of this study might only be relevant to Schatzker type II fractures treated as described. 
Table V. Plain radiological measurements of reduction and proximal tibial morphology in the seven patients at 52 weeks

\begin{tabular}{cllll}
\hline & \multicolumn{2}{l}{ Plain radiological measures } & & \\
\cline { 2 - 5 } & Fracture depression $(\mathbf{m m})$ & $\begin{array}{l}\text { Increase in tibial condylar } \\
\text { width }(\mathbf{m m})\end{array}$ & $\begin{array}{l}\text { Medial proximal tibial } \\
\text { angle }\left(^{\circ}\right)\end{array}$ & $\begin{array}{l}\text { Posterior proximal tibial } \\
\text { angle }\left({ }^{\circ}\right)\end{array}$ \\
\hline Patient & & & 89 & 82 \\
1 & 0 & 2 & 87 & 83 \\
2 & 0 & 0 & 86 & 81 \\
3 & - & 1 & 87 & 79 \\
4 & 0 & 2 & 86 & 83 \\
5 & 0 & -2 & 87 & 80 \\
6 & 0 & 3 & 89 & 82 \\
7 & 0 & 0 & 87 & $81.4(80.0$ to 82.8$)$ \\
Median & 0 & 1 & $87.3(-0.69$ to 2.41$)$ \\
Mean $\left(95 \% \mathrm{Cl}^{*}\right)$ & 0 & $0.86(-0.69$ to 2.41$)$ & & 8 \\
\hline
\end{tabular}

* $\mathrm{Cl}$, confidence interval

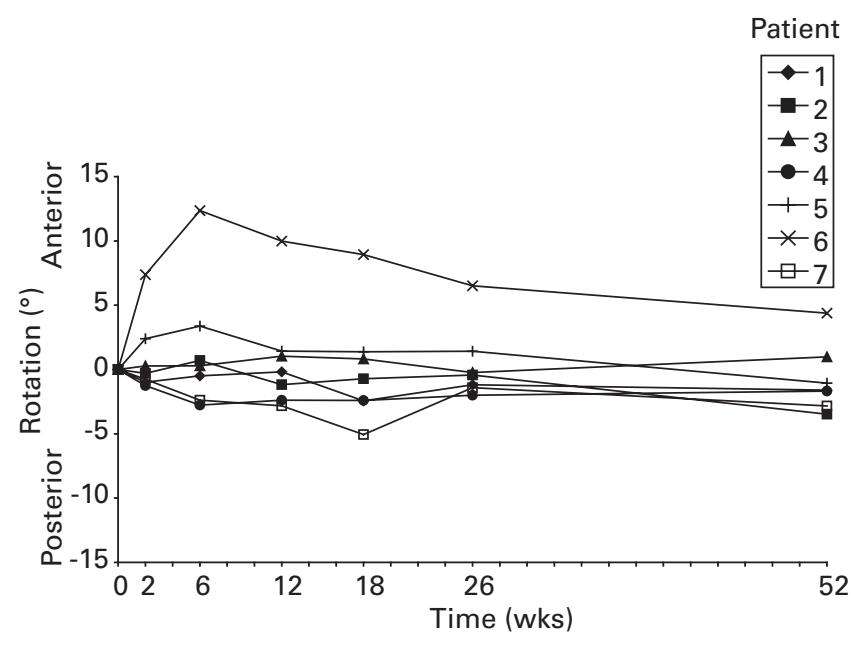

Fig. 5 a

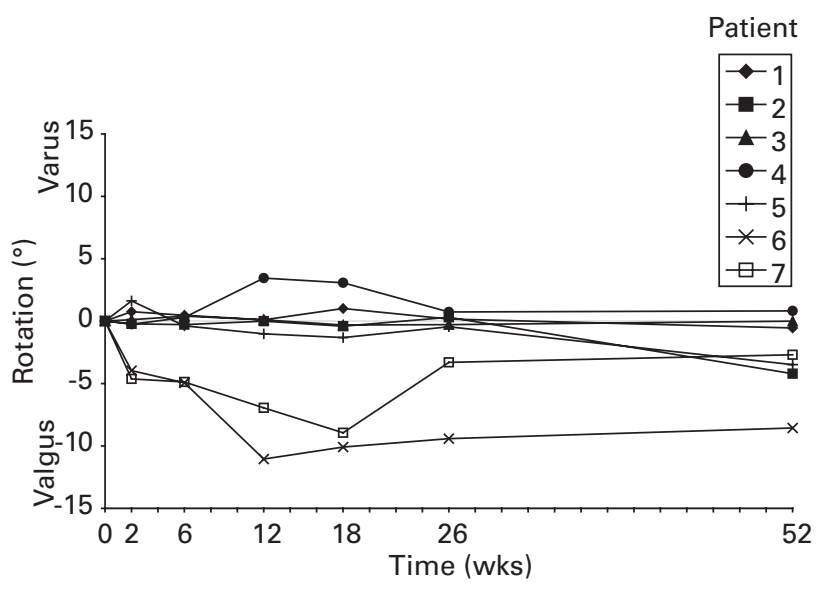

Fig. $5 b$

Graphs showing the overall fracture fragment rotation over time in each patient in a) the $x$-axis (anteroposterior tilt) and b) the $z$-axis (varus-valgus tilt).

Based on manual measurements on plain radiographs, the currently accepted limits of tibial plateau fracture fragment migration thought not to affect clinical outcomes are up to $3 \mathrm{~mm}$ in the craniocaudal direction and $5 \mathrm{~mm}$ in the mediolateral direction. ${ }^{10,11}$ The RSA-measured craniocaudal migration of the fracture fragment corresponds to the articular fracture depression, or step, measured on standard radiographs. Using standard radiographs, no depression was detected, compared to a maximum migration of $1.64 \mathrm{~mm}$ found using RSA. Comparison of these data highlights the lack of sensitivity of plain radiological measures, although in this series of successfully treated Schatzker fractures the measurements made on plain radiographs were sufficient to confirm the maintenance of reduction and proximal tibial morphology.

Based on the results of a cadaver model of simple tibial plateau fractures, an incongruency of articular fragments of $0.5 \mathrm{~mm}$ or less did not generate increased pressures on the surrounding uninjured cartilage, with significant increases occurring only after displacement exceeded $1.5 \mathrm{~mm} .{ }^{22}$ In this RSA study, the mean incongruency was $0.34 \mathrm{~mm}$, with a maximum caudal displacement of $1.64 \mathrm{~mm}$, which suggests that in this cohort there will be a low probability of the development of osteoarthritis. However, only with long-term follow-up will we be able to monitor any development of osteoarthritis.

With RSA and differentially loaded RSA, further studies will be important to redefine the acceptable limits of displacement of fracture fragments under load and migration over time. Of note is that the former, as opposed to measurements made using plain radiographs, allows the identification of angular displacement of the relatively small fracture fragments. However, the importance of such displacements is yet to be defined and cannot be correlated with any measurements made on plain radiographs. In one of our patients (patient 6) the fracture fragment rotated $9.99^{\circ}$ in the sagittal plane and $-11.8^{\circ}$ in the coronal plane 12 weeks after fixation, but there was 
only $-1.15 \mathrm{~mm}$ of craniocaudal and $0.38 \mathrm{~mm}$ of mediolateral translation. Although at the 52 -week follow-up the same fragment's rotation had decreased to $4.3^{\circ}$ in the sagittal plane and $-8.58^{\circ}$ in the coronal plane, the measurements at 12 weeks demonstrate that the fragment was unstable in the first three months after treatment, which indicates the limitation of the method of internal fixation. A post-operative CT scan in this patient confirmed that the central fragment of the fracture was stabilised by only one of the two screws used. In their biomechanical study of lateral tibial plateau fracture fixation, Karunakar et $\mathrm{al}^{2}$ emphasised that, in vitro, screws and plates are positioned where required by the fracture pattern in every case. In vivo, however, it is possible that once the fracture has been reduced, the surgeon cannot easily visualise the reduced fragments and a less than ideal screw placement may occur.

This study provides new RSA data that support the efficacy of immediate partial post-operative weight-bearing in the management of Schatzker type II fractures of the lateral tibial plateau. Favourable results have been reported after immediate partial weight-bearing of $15 \mathrm{~kg}$ in 12 of 14 patients with bicondylar tibial plateau fractures fixed with medial and lateral plating, ${ }^{7}$ and after immediate partial weight-bearing up to $25 \mathrm{~kg}$ in 86 of 102 cases of all types of tibial plateau fracture fixed using a range of methods. ${ }^{8}$ In both studies, plain radiographs were used to assess fracture reduction and maintenance of reduction. Ryd and Toksvig-Larsen ${ }^{23}$ used RSA to measure migration of fracture fragments over time in five tibial plateau fractures treated with screw fixation and plaster immobilisation and non-weight-bearing for three months. The migration pattern during healing was very similar to that in our study, despite our patients commencing partial weight-bearing immediately after surgery.

Using differentially loaded RSA, we have shown that all the central fracture fragments moved minimally under controlled static weight-bearing loads in an elastic manner, confirming successful fixation by the buttress plate and screws. The stiffening over time of the tibial plateau/ internal fixation construct under the loads measured suggests progressive healing of these fractures. Immediately post-operatively, the stability of the fracture fragments when loaded relies entirely on adequate internal fixation. A subchondral raft of screws and a buttress plate in Schatzker type II fractures can withstand loads of up to $35 \mathrm{~kg}$ two weeks after fixation. The fractured bone-internal fixation construct was shown to be able to withstand body weight by six weeks after internal fixation. This in vivo investigative technique offers great potential for comparing the effectiveness and outcome of different methods of fixation, and of post-operative rehabilitation regimes.
This study was funded by research grants from the Australian Orthopaedic Association and the Royal Adelaide Hospital Research Foundations. The radiostereometric analysis cages were funded by Zimmer Pty Ltd, Frenchs Forest, Australia. The patient-lifter used during examination was funded by Smith \& Nephew Pty Ltd, North Ryde, Australia.

No benefits in any form have been received or will be received from a commercial party related directly or indirectly to the subject of this article.

\section{References}

1. Schatzker J, McBroom R, Bruce D. The tibial plateau fracture: the Toronto experience 1968-1975. Clin Orthop 1979;138:94-104.

2. Karunakar MA, Egol KA, Peindl R, et al. Split depression tibial plateau fractures: a biomechanical study. J Orthop Trauma 2002;16:172-7.

3. MusahI V, Tarkin I, Kobbe P, et al. New trends and techniques in open reduction and internal fixation of fractures of the tibial plateau. J Bone Joint Surg [Br] 2009;91-B:426-33.

4. Whittle AP. Fractures of the lower extremity. In: Canale ST, Beaty JH, eds. Campbell's operative orthopaedics. 11th ed. Vol. 3. Philadelphia: Mosby Elsevier, 2007:3085-236.

5. Cole P, Levy B, Watson JT, Schatzker J. Tibial plateau fractures. In: Browner BD, Jupiter JB, Levine AM, Trafton PG, Krettek C, eds. Skeletal trauma: expert consultation. Fourth ed. Vol. 2. Philadelphia: Saunders Elsevier, 2008:2201-87.

6. Egol KA, Koval KJ. Fractures of the tibial plateau. In: Chapman MW, Szabo RM, Marder R, et al, eds. Chapman's orthopaedic surgery. Third ed. Vol. 1. Philadelphia: Lippincott Williams \& Wilkins, 2001:737-54.

7. Eggli S, Hartel MJ, Kohl S, et al. Unstable bicondylar tibial plateau fractures: a clinical investigation. J Orthop Trauma 2008;22:673-9.

8. Russell TA, Leighton RK; Alpha-BSM Tibial Plateau Fracture Study Group. Comparison of autogenous bone graft and endothermic calcium phosphate cement for defect augmentation in tibial plateau fractures: a multicenter, prospective, randomized study. J Bone Joint Surg [Am]2008;90-A:2057-61.

9. Barei DP, Nork SE, Mills WJ, Henley MB, Benirschke SK. Complications associated with internal fixation of high-energy bicondylar tibial plateau fractures utilizing a two-incision technique. J Orthop Trauma 2004;18:649-57.

10. Honkonen SE. Indications for surgical treatment of tibial condyle fractures. Clin Orthop 1994;302:199-205

11. Honkonen SE, Järvinen MJ. Classification of fractures of the tibial condyles. J Bone Joint Surg [Br] 1992;74-B:840-7.

12. Borrelli J Jr, Goldfarb C, Catalano L, Evanoff BA. Assessment of articular fragment displacement in acetabular fractures: a comparison of computerized tomography and plain radiographs. J Orthop Trauma 2002;16:449-56.

13. Cole RJ, Bindra RR, Evanoff BA, et al. Radiographic evaluation of osseous displacement following intra-articular fractures of the distal radius: reliability of plain radiography versus computed tomography. J Hand Surg Am 1997;22:792-800.

14. Liow RY, Birdsall PD, Mucci B, Greiss ME. Spiral computed tomography with twoand three-dimensional reconstruction in the management of tibial plateau fractures. Orthopedics 1999;22:929-32.

15. Martin J, Marsh JL, Nepola JV, et al. Radiographic fracture assessments: which ones can we reliably make? J Orthop Trauma 2000;14:379-85.

16. Solomon LB, Stevenson AW, Callary SA, et al. The accuracy and precision of radiostereometric analysis in monitoring tibial plateau fractures. Acta Orthop 2010;81:487-94

17. Chehade MJ, Solomon LB, Callary SA, et al. Differentially loaded radiostereometric analysis to monitor fracture stiffness: a feasibility study. Clin Orthop 2009;567:1839-47.

18. Paley D, Herzenberg JE, Tetsworth K, McKie J, Bhave A. Deformity planning for frontal and sagittal plane corrective osteotomies. Orthop Clin North Am 1994;25:425-65.

19. Paley D. Normal lower limb alignment and joint orientation. In: Principles of deformity correction. Berlin: Springer, 2002:1-18.

20. Wright TM, Li S. Biomaterials. In: Buckwalter JA, Einhorn TA, Simon SR, eds. Orthopaedic basic science: biology and Bbomechanics of the musculoskeletal system. Second ed. Rosemont: American Academy of Orthopaedic Surgeons, 2000:181-215.

21. Downing MR, Ashcroft PB, Johnstone AJ, et al. Assessment of inducible fracture micromotion in distal radial fractures using radiostereometry. J Orthop Trauma 2008;22(Suppl):96-105.

22. Brown TD, Anderson DD, Nepola JV, et al. Contact stress aberrations following imprecise reduction of simple tibial plateau fractures. J Orthop Res 1988;6:851-62.

23. Ryd L, Toksvig-Larsen S. Stability of the elevated fragment in tibial plateau fractures: a radiographic stereophotogrammetric study of postoperative healing. Int Orthop 1994;18:131-4. 